\title{
Experience With Safe Manual Memory-Management in Cyclone
}

\author{
Michael Hicks \\ University of Maryland \\ Harvard University
}

\author{
Dan Grossman \\ University of Washington
}

\author{
Trevor Jim \\ AT\&T Labs Research
}

\begin{abstract}
The goal of the Cyclone project is to investigate type safety for low-level languages such as C. Our hardest challenge has been providing programmers control over memory management while retaining type safety. This paper reports on our experience trying to integrate and effectively use two previously proposed, type-safe memory management mechanisms: statically-scoped regions and unique pointers. We found that these typing mechanisms can be combined to build alternative memory-management abstractions, such as reference counted objects and arenas with dynamic lifetimes, and thus provide a flexible basis. Our experience-porting $\mathrm{C}$ code and building new applications for resource-constrained systems-confirms that experts can use these features to improve memory footprint and sometimes to improve throughput when used instead of, or in combination with, a conservative garbage collector.
\end{abstract}

\section{Introduction}

Low-level languages such as $\mathrm{C}$ provide a degree of control over space, time, and predictability that highlevel languages such as Java do not. But the lack of type-safety for $\mathrm{C}$ has led to many failures and security problems. The goal of our research is try to bring the "Mohammad of type safety" to the "mountain of existing C code."

To that end, we have been developing Cyclone, a type-safe dialect of C [21]. Cyclone uses a combination of programmer-supplied annotations, an advanced type system, a flow analysis, and run-time checks to ensure that programs are type safe. When we started the project, we relied solely on heap allocation and the Boehm-Demers-Weiser (BDW) conservative garbage collector (GC) to recycle memory safely. BDW provides convenient interoperability with legacy libraries and makes it easy to support polymorphism without needing run-time type tags.

While the BDW collector provides convenience, it does not always provide the performance or control needed by low-level systems applications. In previous work, we described an integration of BDW with type-safe stack allocation and LIFO arena allocation. A region-based, type-and-effect system based upon the work of Tofte and Talpin [29] ensured safety while providing enough polymorphism for reusable code to operate over data allocated anywhere.

In practice, we found that supporting stack allocation was crucial for good performance, and our system was able to infer most region annotations for porting legacy $\mathrm{C}$ code that used stack allocation [13]. We found that LIFO arenas were useful when callers know object lifetimes but only callees can determine object sizes. Unfortunately, LIFO arenas suffer from several well-known limitations that we encountered repeatedly. In particular, they are not suited to computations such as server and event loops.

Since then, we have explored the integration of unique pointers into our memory management framework. Our unique pointers are closely related to typing mechanisms suggested by other researchers, including linear types [30], ownership types [8], alias types [27], and capability types [31]. The critical idea with all of these proposals is to make it easy to track locally the state of an object by forbidding uncontrolled aliasing. In Cyclone, a value with a unique-pointer type is guaranteed to be the only (usable) reference to an object. Such objects can be deallocated by the programmer at any time, and a modular flow analysis is used to ensure that the dangling pointer cannot be dereferenced in the rest of the computation.

Unique pointers are not a novel idea, but we found 
many challenges to implementing them in a fullscale safe language, as they interact poorly with other features such as exceptions, garbage collection, type abstraction, the address-of operator, undefined evaluation order, etc. To our knowledge, no one has attempted to address all of these features in a full-scale language implementation.

On the other hand, we found great synergies in the combination of uniqueness and regions. In particular, we were able to use the LIFO region machinery to support a form of "borrowed" pointers [7], which goes a long way in relaxing the burdens of uniqueness. We were also able to use unique pointers as capabilities for building further memory-management abstractions. In particular, we used unique pointers to control access to a form of dynamically-scoped arenas [16], and for building reference-counted objects and arenas.

In this paper, we briefly describe our support for unique pointers and the extensions they enable; our technical report describes this support in greater detail [19]. We then discuss our experience using these facilities to build or port a few target applications, including a multimedia overlay network, a small web server, a Scheme interpreter, an ftp server, and an image-manipulation program. Most of the applications were chosen because they are structured as (infinite) loops with loop-carried state and are thus not well-suited for LIFO arenas. Furthermore, we feel that these applications are representative for resource-limited platforms, such as cell-phones or embedded systems, where space is at a premium. In most of these applications, we were able to reduce if not eliminate the need for garbage collection. We also saw dramatic improvements in working-set size, and for at least one application an improvement in throughput.

Thus, the contributions of this paper are two-fold:

1. We show that the addition of unique pointers to a region-based language provides a flexible basis for building type-safe, manual memory management, which can complement or replace GC.

2. We confirm that the resource requirements of some important applications can be significantly improved through type-safe, manual memory management.

\section{Regions in Cyclone}

All of the memory management facilities in Cyclone revolve around regions. A region is a logical container for objects that obey some memory management discipline. For instance, a stack frame is a region that holds the values of the variables declared in a lexical block, and the frame is deallocated when control-flow exits the block. As another example, the garbage-collected heap is another region, whose objects are individually deallocated by the collector.

Each region in a program is given a compile-time name, either explicitly by the programmer or implicitly by the compiler. For example, the heap region's name is ' $\mathrm{H}$ and the region name for a function foo's stack frame is ' $f \circ o$. If ' $r$ is the name of a region, and an object $o$ with type $\mathrm{T}$ is allocated in ' $r$, then the type of a pointer to $O$ is written $\mathrm{T} *$ ' $r$.

To ensure that programs never dereference a pointer into a deallocated region, the compiler tracks a conservative approximation of (a) the regions into which a pointer can point, and (b) the set of regions that are still live at each program point. This is implemented using a type-and-effects system in the style of Tofte and Talpin [29].

Cyclone supports region polymorphism, which lets functions and data structures abstract over the regions of their arguments and fields. By default, Cyclone assumes that pointer arguments to functions live in distinct regions, and that all of these regions are live on input. A unification-based algorithm is used to infer instantiations for region-polymorphic functions and the regions for local variables. This drastically cuts the number of region annotations needed for programs. Cyclone also supports region subtyping based on region lifetimes, which combines with region polymorphism to make for an extremely flexible system. In practice, we have found few (bugfree) examples where stack-allocation could not be easily ported from $\mathrm{C}$ to Cyclone.

\subsection{LIFO Arenas}

The basic region system we have described easily supports a form of arenas that have stack-like lastin-first-out (LIFO) lifetimes, but also support dynamic allocation. A LIFO arena is introduced with a lexically-scoped declaration: 


\section{$\{$ region $\langle$ ' $r\rangle h ; \ldots\}$}

Here, $\mathrm{h}$ is a region handle that can be used to allocate in the newly introduced region ' $r$. Calling the primitive $r m a l l o c(h, \ldots)$ allocates space within region ' $r$. When the declaring lexical scope concludes, the handle and the contents of the arena are deallocated.

We have found that arenas work well for situations where the caller does not know how much space to pre-allocate on its stack frame. Even so, we found that the LIFO restriction on arena lifetimes was often too limiting. That is, we often wished that we could deallocate the arena before the end of its scope. This was particularly problematic for loops: If one pushes the arena declaration inside the loop then a fresh arena is created and destroyed for each iteration. Thus, no data can be carried from one iteration to the next, unless they are copied to an arena declared outside the loop. But then all of the data placed in an outer arena would persist until the entire loop terminates. For loops that do not terminate, such as a server request loop or event loop, this is a disaster as the LIFO restriction can lead to unbounded storage requirements.

\section{Unique Pointers}

Very often the limitations of stack allocation and LIFO arenas can simply and conveniently be overcome by using GC. However, GC may not always be compatible with the performance needs of an application. In particular, applications such as embedded systems and network servers sometimes require bounds on space consumption, pause times, or throughput that may be hard to achieve with GC. Therefore, we decided to extend Cyclone with a suite of mechanisms that would permit manual object deallocation without imposing a LIFO restriction. We emphasize that our goal is not necessarily to eliminate $\mathrm{GC}$, but rather to provide programmers with better control over tuning the space and time requirements of their programs.

In general, ensuring that manual deallocation is safe requires very precise information regarding which pointers may alias other pointers. Though there are impressive analyses that compute such aliasing information, they usually require wholeprogram analysis to achieve any level of accuracy.
An alternative solution that has been proposed many times is to restrict or avoid aliasing altogether, so that reasoning about type-states can be done locally. One extreme is to require that pointers which are deallocated be unique (i.e., have no aliases.) In what follows, we briefly describe how we have incorporated unique pointers into Cyclone, enabling support for per-object deallocation, reference-counting, and arenas with dynamic lifetimes.

To distinguish unique pointers from heap-, stack-, or arena-allocated pointers, we use a special region name ' $U$. A pointer value with type $T *$ ' $U$ is created by calling malloc and can be deallocated via free at any time.

We use an intraprocedural, flow-sensitive, pathinsensitive analysis to track when a unique pointer becomes consumed, in which case the analysis rejects a subsequent attempt to use the pointer. We chose an intraprocedural analysis to ensure modular checking and a path-insensitive analysis to ensure scalability. To keep the analysis simple, a copy of a unique pointer (e.g., in an assignment or function call) is treated as consuming the pointer. This ensures that there is at most one usable alias of a unique pointer at any program point. Here is an example:

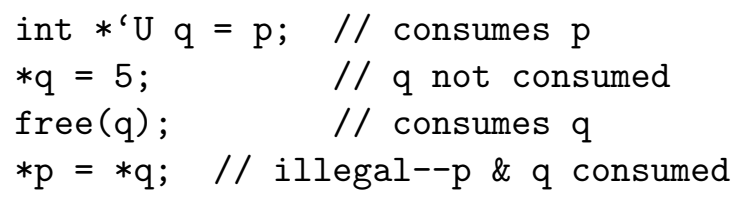

The first assignment aliases and consumes $p$, while the call to free consumes q. Therefore, attempts to dereference $p$ or $q$ are illegal. Dereferencing a unique pointer does not consume it since it does not copy the pointer, as the first dereference of q shows.

At join points in the control-flow graph, our analysis conservatively considers a value consumed if there is an incoming path on which it is consumed. For instance, if $\mathrm{p}$ is not consumed and we write:

$$
\text { if }(\operatorname{rand}()) \text { free }(p) \text {; }
$$

then the analysis treats $p$ as consumed after the statement. In this situation, we issue a warning that $p$ might leak, since the type-states do not match. Fortunately, we can link in the garbage collector to ensure the object is reclaimed. We considered making this an error as in Vault [9], but found that exception handlers and shared unique pointers (described below) 
generated too many false alarms. Thus, we settle for a warning and can rely upon the GC as a safety net.

We allow unique pointers to be placed in nonunique objects (e.g., a global variable or heapallocated object) that might have multiple aliases. To ensure that unique pointers remain unique, we must somehow limit access through these paths. In most systems, reading a unique pointer is treated as a destructive operation that overwrites the original copy with NULL, so as to preserve the uniqueness invariant. In Cyclone, we have pointer types that do not admit NULL as a value, so destructive reads are not always an option. Therefore, we provide an explicit swap operation (":=:") that allows one to swap one unique object for another (including NULL where permitted.) Though notationally less convenient than a destructive read, we found that programming with swaps made us think harder about where NULL-checks were needed, and helped eliminate potential run-time exceptions.

Finally, we remark that placing unique pointers in non-unique objects can lead to subtle "leaks". For instance, there is no guarantee that when a unique pointer is passed to free that the object to which it points does not contain live, unique pointers. Similarly, when we write a unique pointer into a shared object (e.g., a global) there is no guarantee that we are not overwriting another live unique pointer.

\subsection{Borrowing Unique Pointers}

Unique pointers make it easy to support explicit deallocation, but they often force awkward coding idioms just to maintain uniqueness. For example, we must forbid using pointer arithmetic on unique pointers, since doing so could allow the user to call free with a pointer into the middle, rather than the front, of an object, confusing the allocator. As another example, we often want to pass a copy of a pointer to a function that does not consume the pointer, and leave the original copy as unconsumed.

Most systems based on uniqueness or ownership have some way of creating "borrowed" pointers to code around these problems. A borrowed pointer is a second-class copy of a unique pointer that cannot be deallocated and cannot "escape". This ensures that if we deallocate the original pointer, we can invalidate all of the borrowed copies. In Cyclone, a pointer is borrowed using an explicit alias declaration, similar to Walker and Watkins' let-region [33], and the LIFO region machinery prevents the borrowed pointer from escaping.

As an example, consider the following code:

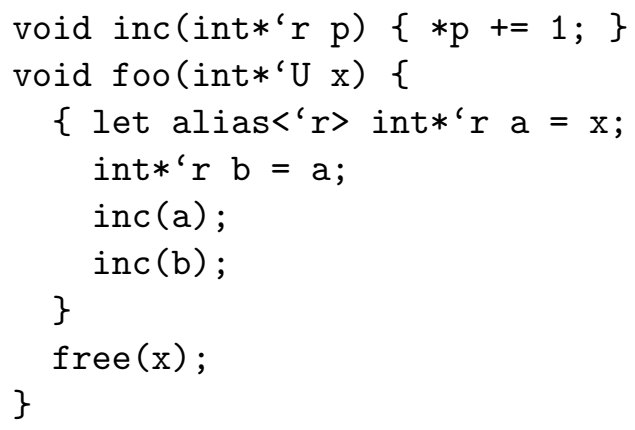

The foo function takes a unique pointer $\mathrm{x}$ as an argument. The declaration "let alias $\langle$ ' $r\rangle$..." introduces a fresh region name, ' $r$, and an alias a for $\mathrm{x}$ that appears to be a pointer into region ' $r$. Within the scope of the alias declaration, we may freely copy a and pass it to functions, just as if it were allocated on the stack. However, throughout the scope of the alias, the variable $\mathrm{x}$ is considered to be consumed. This prevents the unique pointer from being deallocated. At the end of the block, any copies of $x$ will be treated as unusable, since the region has gone out of scope. Thus, no usable aliases can survive the exit from the block, and we can safely restore the type-state of $\mathrm{x}$ to be an unconsumed, unique pointer. In short, regions provide a convenient way to temporarily name unique pointers and track aliasing for a limited scope.

We provide a limited form of alias-inference around function calls to simplify programming and cut down on annotations. For example, we could rewrite foo to be:

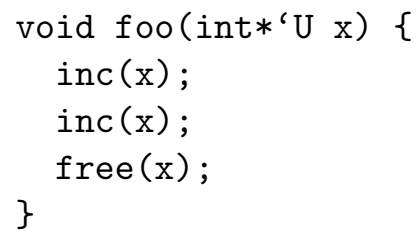

In general, whenever a region-polymorphic function (like inc) is called with a unique pointer as an argument, the compiler will attempt to wrap an alias declaration around the call. This fails to type-check only when the function's return type mentions the 
freshly introduced region name used to identify the unique pointer. In the example above, the compiler successfully wraps each call to inc with a suitable alias declaration for $\mathrm{x}$. In general, the interaction of unique pointers with subtyping and region polymorphism requires some care, but due to space constraints, we refer the reader to our technical report for details [19].

\subsection{Reference Counting}

Even with support for borrowing, unique pointers can be used to build only tree-like data structures with no internal sharing or cycles. While GC or LIFO arenas may be reasonable options for such data structures, another alternative often employed in systems applications is reference-counting. For example, reference-counting is used in COM and the Linux kernel, and is common in $\mathrm{C}++$ and Objective C programs.

We found that we could elegantly support safe reference-counting by building on the discipline of unique pointers. This has two great advantages. First, we introduce almost no new language features, rather only some simple run-time support. Second, the hard work that went into ensuring that unique pointers coexisted with conventional regions is automatically inherited for reference-counted objects.

We define a new reference-counted region ' $\mathrm{RC}$, whose objects, when allocated, are prepended with a hidden reference-count field. As with unique pointers, the flow analysis prevents the user from making implicit aliases. Instead, ' $\mathrm{RC}$ pointers must be copied explicitly by using the alias_refptr function, which increments the reference count and returns a new alias, without consuming the original pointer. In essence, both of the ' $R C$ values serve as explicit capabilities for the same object.

A reference-counted pointer is destroyed by the drop_refptr function. This consumes the given pointer and decrements the object's reference count; if the count becomes zero, the memory is freed.

Thus, treating ' $R C$ pointers as if they were unique forces programmers to manipulate reference counts explicitly. While this is less convenient than automatic reference counting, it requires almost no compiler support. Furthermore, the constraints on unique pointers ensure that an object is never prematurely deallocated, and the flow analysis warns when a pointer is potentially "lost." In addition, we can use the alias construct to borrow a reference-counted pointer to achieve a form of explicit, deferred reference counting. Thus, the programmer has complete control over where reference counts are manipulated.

\subsection{Dynamic Arenas}

Coming full circle, we found we could use unique pointers to provide a more flexible form of arenas that avoids the LIFO lifetime restriction. The basic idea is to use a unique pointer as a capability or "key" for the arena. Accessing the arena requires possession of the key, as does deallocating the arena. Since the key is represented as a unique pointer and is consumed when the arena is destroyed, the arena can no longer be accessed.

Rather than requiring the key be presented on each allocation or pointer-dereference into the arena, we provide a lexically-scoped open construct that temporarily consumes the key and allows the arena to be freely accessed within the scope of the open. The key is then given back upon exit from the scope.

Clearly, open and alias are related. Both provide a way to temporarily "pin" something and give it a name for a particular scope. In the case of alias, a single object is being pinned, whereas in the case of open, an arena is being pinned. Pinning prevents the object(s) from being deallocated throughout the scope, and the region name is used to prevent pointers to the object(s) from escaping the scope. Thus, while we have found lexically-scoped, LIFO arenas of limited use, lexically-scoped region names have proven invaluable for making unique pointers and dynamic arenas work well in practice.

Finally, we have generalized arenas further by supporting reference-counted arenas, where ' $\mathrm{RC}$ pointers are used as keys instead of unique pointers. Thus, the addition of unique pointers to our region framework gives us a number of memory management options. In the following section, we describe our experience trying to use these facilities in various applications. 


\begin{tabular}{|c|c|c|c|c|c|}
\hline \multirow[t]{2}{*}{ Program } & \multirow[t]{2}{*}{ Description } & \multicolumn{3}{|c|}{ Non-comment Lines of Code } & \multirow{2}{*}{$\begin{array}{l}\text { Manual } \\
\text { mechs }\end{array}$} \\
\hline & & $\mathrm{C}$ & Сус & Cyc (+manual) & \\
\hline$\overline{\text { Boa }}$ & web server & 5217 & $\pm 284(5 \%)$ & $7 \pm 91(1 \%)$ & $\overline{\mathrm{U}(\mathrm{D})}$ \\
\hline BetaFTPD & ftp server & 1146 & $\pm 191(16 \%)$ & $\pm 225(21 \%)$ & UR \\
\hline Epic & image compression utility & 2123 & $\pm 217(10 \%)$ & $\pm 114(5 \%)$ & UL \\
\hline Kiss-FFT & fast Fourier transform routine & 453 & $\pm 73(16 \%)$ & $\pm 20(4 \%)$ & \\
\hline MediaNet & streaming data overlay network & & 8715 & $\pm 320(4 \%)$ & URLD \\
\hline CycWeb & web server & & & 667 & \\
\hline CycScheme & scheme interpreter & & & 2523 & ULD \\
\hline
\end{tabular}

Table 1: Benchmark Programs

\section{Applications}

Table 1 describes the benchmark programs with which we experimented. For programs we ported from C (Boa, BetaFTPD, Epic, and Kiss-FFT) it shows the non-comment lines of code of the original program, and then the changes due to porting to $\mathrm{Cy}$ clone to use the GC, and then the additional changes needed to use manual mechanisms. The other programs (MediaNet, CycWeb, and CycScheme) were written directly in Cyclone. The final column indicates which manual mechanisms we used. For all programs other than MediaNet, we eliminated the need for GC entirely. We describe the program and the coding process below. Performance experiments for these programs are presented in the next Section.

\subsection{Porting Experience}

The process of porting from $\mathrm{C}$ to Cyclone is made easiest by placing all dynamically-allocated data in the heap region and letting BDW take care of recycling the data. Most of the changes involve differences between $\mathrm{C}$ and Cyclone that are not memorymanagement related, such as introducing fat pointer annotations. To take advantage of the new manual facilities, we roughly performed two actions. First, we distinguished between those data whose lifetimes are scoped from those whose are not. Second, for those data structures with a non-scoped lifetime, we identified their aliasing behavior to determine which mechanism to use.
Objects with Scoped Lifetime When data structures have scoped lifetimes, we can either allocate them in a LIFO arena, or we can allocate them in the unique region, and use the alias construct to allow temporary aliasing until they can be freed. For example, in both Epic and Kiss-FFT, we merely had to change a declaration from something like

$$
\text { T* q_pyr }=\operatorname{malloc}(\ldots) \text {; }
$$

to instead be

$$
\mathrm{T} * \text { 'U q_pyr }=\operatorname{malloc}(\ldots) ;
$$

All alias statements were inferred automatically when calling subroutines that wished to alias the array. For Epic, we also used a LIFO arena to store a Huffman compression tree that was used during the first phase of the compression. This required changing the prototypes of the creator functions to pass in the appropriate arena handle, in addition to adding various region annotations.

Objects with Dynamic Lifetime If we wish to manually manage a data structure using unique pointers, it cannot require aliases. For example, Boa stores the state of each request in a request structure, illustrated in Figure 1. Because these form a doubly-linked list, we cannot use unique pointers. Even if we were to remove the next and prev fields and store request objects in a separate (nonunique) list, we could not uniquely allocate requests because they contain internal aliases. For example, the header field identifies the HTTP header in an internal buffer. 


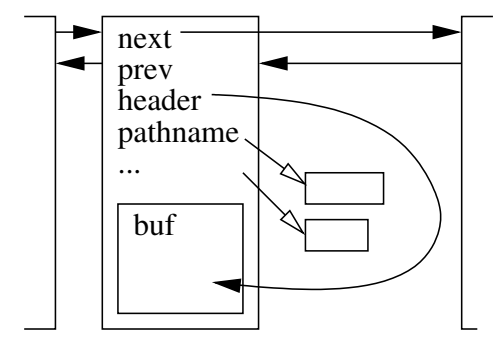

Figure 1: Request data structure in Boa

In the case of Boa, request objects are managed by a custom allocator. Throughout its lifetime, a request is moved between a blocked queue and a ready queue, and when complete, the request is moved onto a free list to be reused. Therefore, we can continue to use this allocator and simply heap-allocate the requests since they will never be freed by the system allocator. Some internal elements of the request, such as the pathname (shown with open-headed arrows in the figure) were not aliased internally, so they could be uniquely allocated and freed when the request was complete. We also experimented with a version that used dynamic arenas for requests instead of the custom allocator, but found that this adversely affected throughput. We remark that this alternative only required changes to the topmost request management routines; the internal request processing routines could remain the same.

BetaFTPD also used doubly-linked lists, one for transfers in progress, and one for open sessions. Furthermore, there were cross-links between session and transfer objects. Thus, reference-counted pointers seemed a natural fit. As can be seen from Table 1, this required $21 \%$ of the code to be changed, significantly more than the other ports. The reason is that all reference-counts are managed manually, so we had to insert many calls to alias_refptr and drop_refptr along with the addition of annotations. While largely straightforward, we were forced to spend some time tracking down memory leaks that arose from failing to decrement a count. The warnings issued by the compiler were of little help, since there were too many false positives. However, we remark that the original program had a space leak along a failure path that we were able to find and eliminate.

\subsection{Cyclone Applications}

In addition to porting $\mathrm{C}$ programs, we have written three Cyclone programs from scratch that use our manual mechanisms. Here we describe which mechanisms we used and why.

\subsubsection{CycWeb}

CycWeb is a simple, space-conscious web server that supports concurrent connections using non-blocking I/O and an event library in the style of libasync [23] and libevent [26]. The event library lets users register callbacks for I/O events. A callback consists of a function pointer and an explicit environment that is passed to the function when it is called. The event library uses polymorphism to allow callbacks and their environments to be allocated in arbitrary regions. This generality is not overly burdensome: of 260 lines of code, we employ our swap operator only 10 times across 10 functions, and never use the alias primitive explicitly. The entire web server uses swap 16 times and an explicit alias 5 times.

For the rest of the application, we also chose to use unique pointers. When a client requests a file, the server allocates a small buffer for reading the file and sending it to the client in chunks (default size is $1 \mathrm{~KB}$ ). Callbacks are manually freed by the event loop when the callback is invoked (they must be reregistered if an entire transaction is not completed); each callback is responsible for freeing its own environment, if necessary. As we see in the next section, this design allows the server to be reasonably fast while consuming very little space.

\subsubsection{MediaNet}

MediaNet [20] is an overlay network whose servers forward packets according to a reconfigurable DAG of operations, where each operation works on the data as it passes through. For better performance, we eschew copying packets between operations unless correctness requires it. However, the dynamic nature of configurations means that both packet lifetimes and whether packets are shared cannot be known statically.

We store the DAG of operations inside a dynamic arena, creating a new arena with each configuration. We use a data structure called a streambuff 


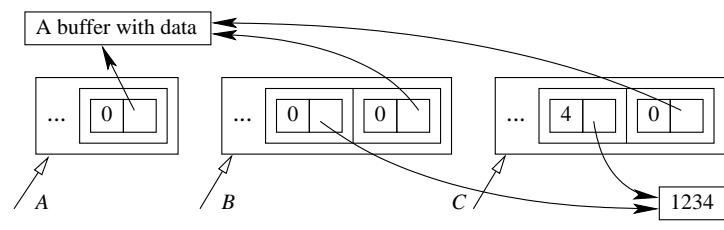

Figure 2: Pointer graph for three streambuffs

for each packet, similar to a Linux skbuff, which consists of a unique pointer to an array of referencecounted buffers, each paired with an offset. Whenever a packet must be shared, a new streambuff is created, whose array points to the same databuffers as the original (after increasing their reference counts). This approach allows for quickly appending and prepending data to a packet, and requires copying packet buffers only when they are both shared and mutated. Finally, we found it convenient to allocate some objects in the heap, such as objects representing connections. This means that we must use the GC to manage these objects.

An example using streambuffs is shown in Figure 2. Here, three individual streambuffs $A, B$, and $C$ share some underlying data; unique pointers have open arrowheads, while reference-counted ones are filled in. This situation could have arisen by (1) receiving a packet and storing its contents in $A$; (2) creating a new buffer $B$ that prepends a sequence number 1234 to the data of $A$; and (3) stripping off the sequence number for later processing (assuming the sequence number's length is 4 bytes). Thus, $C$ and $A$ are equivalent. When we free a streambuff, we decrement the reference counts on its databuffs, so they will be freed as soon as possible.

An earlier version of MediaNet stored all packet and configuration data in the garbage-collected heap, and used essentially the same structures for packet data. One important difference was that databuffs contained an explicit refont field managed by the application to implement copy-on-write semantics. Unfortunately, this approach yielded a number of hard-to-find bugs due to reference count mismanagement. Our language support for reference-counting eliminated the possibility of these bugs, and further let us free the data immediately after its last use. As shown in Table 1, moving to explicit unique pointers and dynamic regions was not difficult; only $4 \%$ of the code had to be changed. The majority of these changes were in a couple of utility files. Out of nearly 9000 non-comment lines, we added 76 annotations, used swap 46 times, and alias 66 times, of which $71 \%$ were automatically inferred.

\subsubsection{CycScheme}

Using a combination of our dynamic arenas and unique pointers, Fluet and Wang [11] have implemented a Scheme interpreter and runtime system in Cyclone. The runtime system includes a copying garbage collector in the style of Wang and Appel [34], written entirely in type-safe Cyclone. All data from the interpreted program are allocated in an arena, and when the collector is invoked, the live data are copied from one arena to another, and the old arena is then deallocated. Since both arenas must be live during collection but their lifetimes are not nested, LIFO arenas would not be sufficient.

Further details on CycScheme's performance and implementation can be found in Fluet and Wang's paper. We simply observe that our system of memory management was flexible enough for this interesting application. In particular, this shows that at least in principle, it is possible for Cyclone programs to code up their own copying garbage collectors tailored for a particular application. However, we remark that the approach is limited to straightforward copying collection and does not accommodate other techniques, including generational techniques or mark/sweep.

\section{Performance Experiments}

To understand the benefit of our proposed mechanisms, we compared the performance of the GC-only versions of our sample applications to the ones using manual mechanisms. Our measurements exhibit two trends. First, we found that elapsed time is similar for the GC and manual versions of the programs. Indeed all of our benchmark programs, other than MediaNet, have execution time performance virtually the same for the GC and non-GC cases. Second, we found that we could significantly reduce memory utilization by making use of manual mechanisms. Moreover, in the case of MediaNet, we also improved its throughput: judicious use of manual 


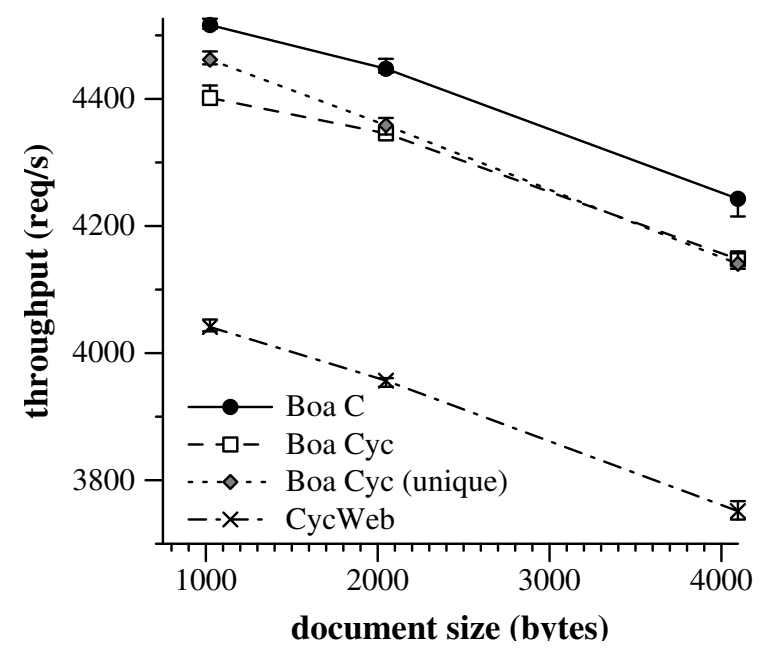

Figure 3: Throughput for CycWeb and Boa

mechanisms significantly reduced the reliance on GC (but did not eliminate it entirely), improving performance.

In this section we carefully discuss the performance of the Boa, CycWeb, and MediaNet servers. We found these to be the most interesting programs from a resource management point of view; measurements for the remaining programs can be found in the Appendix. We ran our performance experiments on a cluster of dual-processor 1.6 GHz AMD Athlon MP 2000 workstations each with 1 GB of RAM and running Linux kernel version 2.4.20-20.9smp. The cluster is connected via a Myrinet switch.

We used Cyclone version 0.8, which can be downloaded from http://www.cs. cornell.edu/ projects/cyclone/. By default, Cyclone uses the Boehm-Demers-Weiser (BDW) conservative garbage collector [5], version $6.2 \alpha 4$, for garbage collection and manual deallocation. BDW uses a marksweep algorithm, and is incremental and generational. We used the default initial heap size and heapgrowth parameters for these experiments. When programs do not need GC, they can be compiled with the Lea general-purpose allocator [22]. Cyclone compiles to $\mathrm{C}$, which then uses gcc version 3.2.2, with optimization level -02 , to create executables.

\subsection{Boa and CycWeb}

We measured web server throughput using the SIEGE web benchmarking tool ${ }^{1}$ to blast Boa with repeated requests from 6 concurrent users for a single file of varying size for 10 seconds. (We chose 6 users because we observed it maximized server performance.) The throughput results are shown in Figure 3-note the non-zero y-axis. This shows three versions of Boa- $\mathrm{C}$, Cyclone using $\mathrm{GC}$, Cyclone without GC (labeled "unique")—and the single version of CycWeb. We plot the median of 15 trials, and the error bars show the quartiles. For Boa, the performance difference between the $\mathrm{C}$ and Cyclone versions is between 2 and 3\%, and the differences between the various Cyclone versions are negligible (often within the range of error or close to it). Thus, for the performance metric of throughput, removing the GC has little payoff. CycWeb is optimized for memory footprint instead of speed, but comes within $10-15 \%$ of Boa in C.

Avoiding GC has greater benefit when considering memory footprint. Figure 4 depicts three traces of Boa's memory usage while it serves $4 \mathrm{~KB}$ pages to 6 concurrent users. The first trace uses GC, while the second two make use of unique pointers. The second (unique+GC) uses BDW as its allocator (thus preventing inadvertent memory leaks), while the third uses the Lea allocator. ${ }^{2}$ The $\mathrm{x}$-axis is elapsed time, while the $y$-axis plots memory consumed. The graph shows memory used by the heap region and by the unique region, as well as the total space reserved by the allocator (i.e., acquired from the operating system).

The working set size of all versions is similar, and is dominated by the heap region since the majority of memory is consumed by the heap-allocated request structures. The GC version's footprint fluctuates as request elements are allocated and collected (each GC, of which there are a total of 33 in this case, is depicted as a vertical line). To ensure reasonable performance, the collector reserves a fair amount of headroom from the OS: $635 \mathrm{~KB}$ in this case. By contrast, the unique versions have far less reserved space, with the Lea allocator having little more than

\footnotetext{
${ }^{1}$ http://joedog.org/siege/

${ }^{2}$ The throughput of both versions is essentially the same, so only one line is shown in Figure 3.
} 


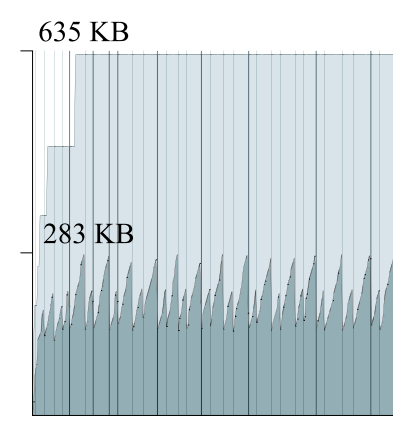

Boa Cyc (GC)

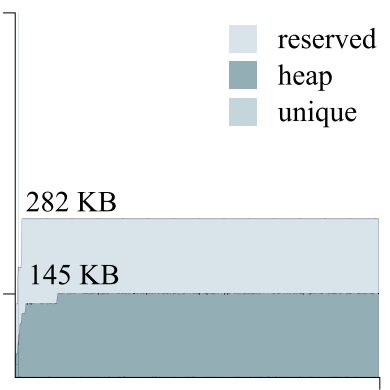

Boa Cyc (unique+GC)

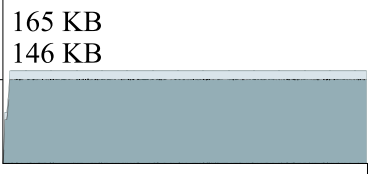

Boa Cyc (unique)

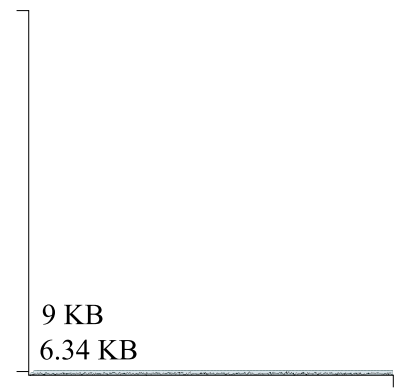

CycWeb

Figure 4: Memory footprint of Cyc Boa versions

that required by the application. We have done memory traces with other heap sizes and levels of concurrent access and found the trends to be similar. Very little data is allocated in the unique region (it is not really visible in the graph) — only about 50 bytes per request. In the GC case, this same data is allocated in the heap, and accumulates until eventually collected.

Turning to $\mathrm{CycWeb}$, which uses only the Lea allocator and no garbage collector, we see that we have succeeded in minimizing memory footprint: the working set size is less than $6.5 \mathrm{~KB}$. This is proportional to the number of concurrent requests-we process at most 6 requests at a time, and allocate a $1 \mathrm{~KB}$ buffer to each request.

\subsection{MediaNet}

All of the versions of Boa perform very little allocation per transaction, thanks to the use of a custom allocator. The benefit of the allocator depends in part on the fact that request objects are uniformlysized: allocations merely need to remove the first element from the list. The same approach would work less well in an application like MediaNet, whose packets vary widely in size (from a tens of bytes to tens of kilobytes). Avoiding excessive internal fragmentation would require managing multiple pools, at which point a general-purpose allocator seems more sensible, which is what we used. However, we found that this choice can lead to significant overhead when using GC.

In a simple experiment, we used the TTCP microbenchmark [25] to measure MediaNet's packetforwarding throughput and memory use for vary-

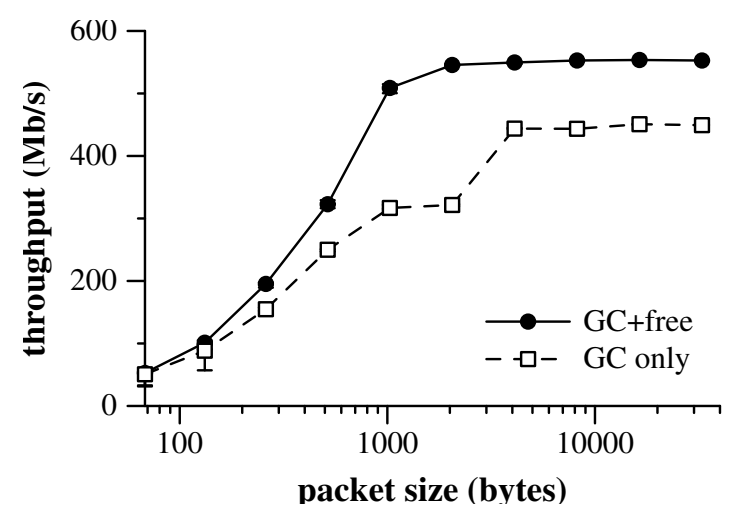

Figure 5: MediaNet throughput

ing packet sizes. We measured two configurations. GC+free is MediaNet built using unique and reference-counted pointers for its packet objects (as described above), while GC only stores all packet objects in the garbage-collected heap.

Figure 5 plots the throughput, in megabits per second, as a function of packet size (note the logarithmic scale). Each point is the median of 21 trials in which 5000 packets are transferred; the error bars plot the quartiles. The two configurations perform roughly the same for the smallest packet sizes, but GC only quickly falls behind as packets reach 256 bytes. Both curves level off at $4 \mathrm{~KB}$ packets, with the $G C+$ free case achieving $23 \%$ better throughput. The odd leveling of the slope in the GC only curve at $2 \mathrm{~KB}$ packets results in a $70 \%$ difference. $^{3}$ This experiment illustrates the benefit of being able to free a packet immediately. While more sophisticated garbage col-

\footnotetext{
${ }^{3}$ We suspect this dip is due to kernel scheduling issues [24].
} 


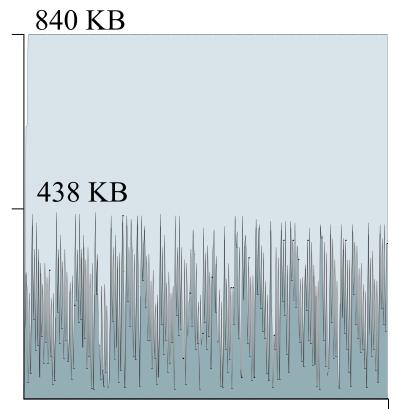

MediaNet (GC only)

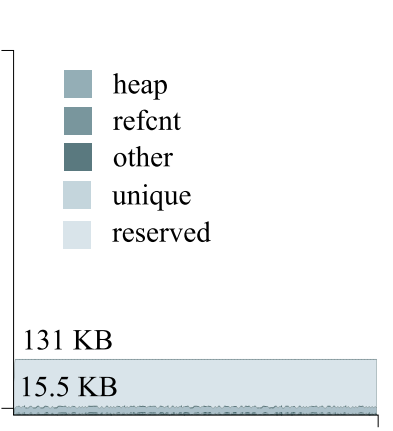

MediaNet (GC+free)

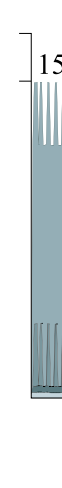

heap 3.66 MB*clocks (82\%)

refcnt $609 \mathrm{~KB}^{*}$ clocks $(13 \%)$

other $98.3 \mathrm{~KB}^{*}$ clocks $(2 \%)$

unique $86.5 \mathrm{~KB} *$ clocks $(1 \%)$

Figure 6: MediaNet memory utilization

lectors could well close the gap, the use of manual mechanisms can only be of help. Moreover, even advanced GC's will do less well when packet lifetimes vary due to user processing in the server; our use of reference-counting allows packets to be shared and freed immediately when no longer of interest.

Figure 6 illustrates the memory usage of MediaNet when forwarding 50,000 $4 \mathrm{~KB}$ packets. This graph has the same format as the graph in Figure 4; it shows the heap, unique, and reference-counted regions, and the dynamic region in which the configuration is stored (labeled "other"). The GC only configuration stores all data in the heap region, which exhibits a sawtooth pattern with each peak roughly coinciding with a garbage collection (there were 553 total on this run). The $G C+$ free configuration uses and reserves far less memory (131 KB as opposed to $840 \mathrm{~KB}$ for reserved memory, and $15.5 \mathrm{~KB}$ as opposed to $438 \mathrm{~KB}$ of peak used memory). There are about $10 \mathrm{~KB}$ of initial heap-allocated data that remain throughout the run, and the reference-counted and unique data never consume more than a single packet's worth of space, since each packet is freed before the next packet is read in. This can be seen in the close-up at the right of the figure. The topmost band is the heap region (the reserved space is not shown), while the feathery band below it is the reference-counted region. Below that is the dynamic region and finally the unique region.

These performance trends are consistent with other studies comparing GC and manual memory management $[35,18]$. What we have shown is that some simple and safe manual mechanisms can comple- ment $\mathrm{GC}$ in attacking problems of memory management. They give programmers more control over the performance of their programs, without undue programming burden, and without need to compromise safety.

\section{Related Work}

The ML Kit [28] implements Standard ML with (LIFO) regions. Type inference is used to automatically allocate data into regions. Various extensions have relaxed some LIFO restrictions [2, 14], but unique pointers have not been considered.

The RC language and compiler [12] provide language support for reference-counted regions in $\mathrm{C}$. However, RC does not prevent dangling pointers to data outside of regions and does not provide the typesafety guarantees of Cyclone.

Work on linear types [30], alias types [27, 32], linear regions [33, 17], and uniqueness types [1] provide important foundations for safe manual memory management on which we have built. Much of this foundational work has been done in the context of core functional languages and does not address the range of issues we have.

Perhaps the most relevant work is from the Vault project $[9,10]$ which also uses regions and linearity. Unique pointers allow Vault to track sophisticated type states, including whether memory has been deallocated. To relax the uniqueness invariant, they use novel adoption and focus operators. Adoption lets programs violate uniqueness by choosing a unique object to own a no-longer-unique object. 
Deallocating the unique object deallocates both objects. Compared to Cyclone's support for unique pointers in non-unique context, adoption prevents more space leaks, but requires hidden data fields so the run-time system can deallocate data structures implicitly. Focus (which is similar to Foster and Aiken's restrict [3]) allows adopted objects to be temporarily unique. Compared to swap, focus does not incur run-time overhead, but the type system to prevent access through an unknown alias requires more user annotations (or a global alias analysis.)

Unique pointers and related alias restrictions have received considerable attention as extensions to object-oriented languages. Clarke and Wrigstad [8] provide an excellent review of related work and propose a notion of "external uniqueness" that integrates unique pointers and ownership types. Prior to this work, none of the analogues to Cyclone's alias allowed aliased pointers to be stored anywhere except in method parameters and local variables, severely restricting code reuse. Clarke and Wrigstad use a "fresh owner" to restrict the escape of aliased pointers, much as Cyclone uses a fresh region name with alias. Ownership types differ from our region system most notably by restricting which objects can refer to other objects instead of using a static notion of accessible regions at a program point.

Little work on uniqueness in OO languages has targeted manual memory management. A recent exception is Boyapati et al.'s work [6], which uses regions to avoid some run-time errors in Real-Time Java programs. As is common, this work uses "destructive reads" (an atomic swap with NULL) and relies on an optimizer to eliminate unnecessary writes of NULL on unique paths. Cyclone resorts to swaps only for unique data in nonunique containers, catching more errors at compile time. Few other projects have used swap instead of destructive reads $[4,15]$. Alias burying [7] eschews destructive reads and proposes using static analysis to prevent using aliases after a unique pointer is consumed, but the details of integrating an analysis into a language definition are not considered.

\section{Conclusions}

Cyclone now supports a rich set of safe memorymanagement idioms beyond garbage collection:

- Stack/regions: works well for lexically-scoped lifetimes.

- Dynamic arenas: works well for aggregated, dynamically allocated data.

- Uniqueness: works well for individual objects as long as multiple references aren't needed within data structures.

- Reference counting: works well for individual objects that must be shared, but requires explicit reference count management.

Programmers can use the best idioms for their application. In our experience, all idioms have proven useful for improving some aspect of performance.

This array of idioms is covered by the careful combination of only two linguistic features: lexically scoped regions and unique pointers. Unique pointers give us the power to reason in a flow-sensitive fashion about the state of objects or arenas and to ensure that safety protocols, such as reference counting, are enforced. Regions work well for stack allocation and give us a way to overcome the burdens of uniqueness for a limited scope.

Nonetheless, there are many open issues that require further research. For instance, a strict, linear interpretation of unique pointers instead of our relaxed affine approach would have helped to avoid the leaks that we encountered and perhaps avoid the need for GC all together. However, we found that the strict interpretation generated too many false type-errors in the presence of exceptions and global data.

Another area where further work is needed is in tools to assist the porting process. We generally found that developing new code in Cyclone was easier because we could start with the invariants for a particular memory management strategy in mind. In contrast, porting legacy code required manually extracting these invariants from the code. Our hope is that we can adapt tools from the alias and shape analysis community to assist programmers in porting applications. 


\section{References}

[1] Peter Achten and Rinus Plasmeijer. The ins and outs of Clean I/O. Journal of Functional Programming, 5(1), 1995.

[2] Alex Aiken, Manuel Fähndrich, and Raph Levien. Better static memory management: Improving region-based analysis of higher-order languages. In PLDI, 1995.

[3] Alex Aiken, Jeffrey S. Foster, John Kodumal, and Tachio Terauchi. Checking and inferring local nonaliasing. In PLDI, 2003.

[4] Henry Baker. Lively linear LISP-look ma, no garbage. ACM SIGPLAN Notices, 27(8), 1992.

[5] Hans-Juergen Boehm and Mark Weiser. Garbage collection in an uncooperative environment. Software - Practice and Experience, 18(9), 1988.

[6] Chandrasekhar Boyapati, Alexandru Sălcianu, William Beebee, and Martin Rinard. Ownership types for safe region-based memory management in real-time Java. In PLDI, 2003.

[7] John Boyland. Alias burying: Unique variables without destructive reads. Software - Practice and Experience, 31(6), 2001.

[8] Dave Clarke and Tobias Wrigstad. External uniqueness is unique enough. In ECOOP, 2003.

[9] Robert DeLine and Manuel Fähndrich. Enforcing high-level protocols in low-level software. In PLDI, 2001.

[10] Manuel Fähndrich and Robert DeLine. Adoption and focus: Practical linear types for imperative programming. In PLDI, 2002.

[11] Matthew Fluet and Daniel Wang. Implementation and performance evaluation of a safe runtime system in Cyclone. In Informal Proceedings of the SPACE 2004 Workshop, 2004. Available at http://www.diku.dk/topps/space2004/ space_final/fluet-wang.pdf.

[12] David Gay and Alex Aiken. Language support for regions. In PLDI, 2001.

[13] Dan Grossman, Greg Morrisett, Trevor Jim, Michael Hicks, Yanling Wang, and James Cheney. Regionbased memory management in Cyclone. In PLDI, 2002.

[14] Niels Hallenberg, Martin Elsman, and Mads Tofte. Combining region inference and garbage collection. In PLDI, 2002.
[15] Douglas Harms and Bruce Weide. Copying and swapping: Influences on the design of reusable software components. IEEE Transactions on Software Engineering, 17(5), 1991.

[16] Chris Hawblitzel. Adding Operating System Structure to Language-Based Protection. $\mathrm{PhD}$ thesis, June 2000.

[17] Fritz Henglein, Henning Makholm, and Henning Niss. A direct approach to control-flow sensitive region-based memory management. In PPDP, 2001.

[18] Matthew Hertz and Emery Berger. Automatic vs. explicit memory management: Settling the performance debate. Technical Report CS TR-04-17, University of Massachussetts Department of Computer Science, 2004.

[19] Michael Hicks, Greg Morrisett, Dan Grossman, and Trevor Jim. Safe and flexible memory management in Cyclone. Technical Report CS-TR-4514, University of Maryland Department of Computer Science, July 2003.

[20] Michael Hicks, Adithya Nagajaran, and Robbert van Renesse. MediaNet: User-defined adaptive scheduling for streaming data. In IEEE OPENARCH, 2003.

[21] Trevor Jim, Greg Morrisett, Dan Grossman, Michael Hicks, James Cheney, and Yanling Wang. Cyclone: A safe dialect of C. In Proceedings of the USENIX Annual Technical Conference, 2002.

[22] Doug Lea. A memory allocator. http://gee.cs . oswego.edu/dl/html/malloc.html.

[23] David Mazières. A toolkit for user-level file systems. In USENIX Annual Technical Conference, 2001.

[24] Jonathan T. Moore, Michael Hicks, and Scott Nettles. Practical programmable packets. In INFOCOM, 2001.

[25] Mike Muuss. The story of TTCP. http://ftp. arl.mil/〜mike/ttcp.html.

[26] Niels Provos. libevent - an event notification library. http://www. monkey. org/ provos/ libevent/.

[27] Fred Smith, David Walker, and Greg Morrisett. Alias types. In ESOP, 2000.

[28] Mads Tofte, Lars Birkedal, Martin Elsman, Niels Hallenberg, Tommy Højfeld Olesen, and Peter Sestoft. Programming with regions in the ML Kit (for version 4). Technical report, IT University of Copenhagen, 2001. 
[29] Mads Tofte and Jean-Pierre Talpin. Region-based memory management. Information and Computation, 132(2), 1997.

[30] Philip Wadler. Linear types can change the world! In Programming Concepts and Methods, 1990. IFIP TC 2 Working Conference.

[31] David Walker, Karl Crary, and Greg Morrisett. Typed memory management in a calculus of capabilities. TOPLAS, 24(4), 2000.

[32] David Walker and Greg Morrisett. Alias types for recursive data structures. In Workshop on Types in Compilation, 2000.

[33] David Walker and Kevin Watkins. On regions and linear types. In ICFP, 2001.

[34] Daniel Wang and Andrew Appel. Type-preserving garbage collectors. In POPL, 2001.

[35] Benjamin G. Zorn. The measured cost of conservative garbage collection. Software - Practice and Experience, 23(7):733-756, 1993.

\section{A Additional Performance Measurements}

In this section we present performance measurements for the benchmarks not considered in Section 5. In general, these benchmarks exhibit the following trends (as mentioned in the body of the paper):

- Using manual memory management mechanisms does not improve the execution time of the program relative to $\mathrm{GC}$.

- Using manual memory management does allow the memory footprint of the program to be reduced.

We measured the performance of each program as follows. For Epic, we used it to compress and decompress a large image file. For Kiss-FFT, we performed 1024 size 10000 FFT's, using the benchmark program provided with the distribution. For BetaFTPD, we used $\mathrm{WGET}^{4}$, a utility for retrieving files from HTTP and FTP servers, to retrieve a 20 KB file via anonymous FTP 1000 times, piping it to /dev/null.

\footnotetext{
${ }^{4}$ http://www.gnu.org/directory/wget.html
}

\section{A.1 Elapsed Time Measurements}

The results of measuring the elapsed time of each benchmark are shown in Table 2. Here we measure the $\mathrm{C}$ and Cyclone versions, with the top three rows considering Cyclone using GC, and the bottom three using manual mechanisms (no GC needed). We also report the performance of Cyclone with and without array bounds checks enabled. Each number reports elapsed time in seconds, and is the median of 21 trials, with \pm referring to the scaled semi-interquartile range (SIQR). The SIQR measures variance, similar to standard deviation, by calculating the distance between the quartiles and scaling it to the median.

For the computationally-intensive Epic and KissFFT programs, we see that Cyclone can be substantially slower than $\mathrm{C}$ due to array bounds checks. While Cyclone does some array-bounds check elimination, this is an area of current work in the compiler. With these particular benchmarks, the problem is the use of pointer arithmetic. Our compiler could eliminate many more checks if we were to restructure the program to use array indexes instead.

For BetaFTPD there is no appreciable difference between the $\mathrm{C}$ and Cyclone versions. To acquire a file via anonymous FTP requires roughly six configuration commands, at which time the client instructs the server to connect back to it on a specified port to send the data. As a result, retrieving a file using anonymous FTP is very much I/O-bound, and quite time-consuming, so there is little concern about the CPU-time or pause-time overhead incurred by garbage collection (or reference-counting, for that matter). On the other hand, BetaFTPD is clearly not well optimized, as each FTP takes $22 \mathrm{~ms}$.

Of most concern to the topic of this paper, we can see that using manual memory management (in this case, unique and reference-counted pointers) did not provide a performance advantage relative to Cyclone using GC when considering elapsed time.

\section{A.2 Memory Footprint Measurements}

Statistics for memory footprint are shown in Table 3. For each benchmark we report the peak memory usage for the data memory and reserved memory, in kilobytes. The first group of numbers are for the GC case, while the last group are for the manual case, 


\begin{tabular}{|l|c|cc|cc|}
\hline Test & C time(s) & \multicolumn{4}{|c|}{ Cyclone time (GC) } \\
& & checked(s) & factor & unchecked(s) & factor \\
\hline Epic & $1.06 \pm 0.00$ & $1.60 \pm 0.00$ & $1.51 \times$ & $1.05 \pm 0.01$ & $0.99 \times$ \\
Kiss-FFT & $1.33 \pm 0.00$ & $3.21 \pm 0.01$ & $2.41 \times$ & $1.30 \pm 0.01$ & $0.98 \times$ \\
BetaFTPD & $2.17 \pm 0.02$ & $2.25 \pm 0.02$ & $1.04 \times$ & $2.22 \pm 0.01$ & $1.02 \times$ \\
\hline
\end{tabular}

Cyclone time (+manual)

\begin{tabular}{|l|c|cc|cc|}
\hline Epic & $1.06 \pm 0.00$ & $1.61 \pm 0.01$ & $1.52 \times$ & $1.06 \pm 0.00$ & $1.00 \times$ \\
Kiss-FFT & $1.33 \pm 0.00$ & $3.22 \pm 0.01$ & $2.42 \times$ & $1.31 \pm 0.00$ & $0.98 \times$ \\
BetaFTPD & $2.17 \pm 0.02$ & $2.24 \pm 0.01$ & $1.03 \times$ & $2.23 \pm 0.02$ & $1.03 \times$ \\
\hline
\end{tabular}

Table 2: Benchmark performance

and we consider the reserved memory for the case when using the BDW collector as the allocator or the Lea allocator. None of the manual versions of these programs require garbage collection.

For Epic there is a memory utilization advantage to the manual case because we are able to free some data early, i.e., during the compression process. On the other hand, the FFT program is set up to only free its memory upon conclusion, so there is no real effect on data footprint, and thus the only benefit is to reduce the about of reserved space by linking in the Lea allocator. The trend for BetaFTPD is similar to that of Boa, shown earlier. In particular, when using garbage collection both the data footprint and the reserved memory required are much higher than for the manual mechanisms. We do not show the memoryconsumption graphs here, but they are essentially the same as Boa (and MediaNet): a sawtooth pattern for the GC case (for a total of 11 GCs during the run), and a smooth trend for the manual case.

\begin{tabular}{|l|rr|rrr|}
\hline Test & \multicolumn{2}{|c|}{ KB Footprint } & \multicolumn{3}{c|}{ KB Footprint } \\
& $\begin{array}{c}\mid \text { (GC) } \\
\text { data }\end{array}$ & resv & data & $\begin{array}{r}\text { resv } \\
\text { (BDW) }\end{array}$ & $\begin{array}{r}\text { resv } \\
\text { (Lea) }\end{array}$ \\
\hline Epic & 17475 & 23400 & 13107 & 15585 & 13128 \\
Kiss-fft & 400 & 725 & 400 & 725 & 402 \\
BetaFTPD & 183 & 356 & 3.3 & 65 & 8 \\
\hline
\end{tabular}

Table 3: Benchmark Memory Footprint 